\title{
A posteriori error analysis of an augmented mixed finite element method for Darcy flow
}

\author{
Tomás P. Barrios ${ }^{*}$ J. Manuel Cascón ${ }^{\dagger}$ and María GonzÁlez J $^{\ddagger}$
}

\begin{abstract}
We develop an a posteriori error analysis of residual type of a stabilized mixed finite element method for Darcy flow. The stabilized formulation is obtained by adding to the standard dualmixed approach suitable residual type terms arising from Darcy's law and the mass conservation equation. We derive sufficient conditions on the stabilization parameters that guarantee that the augmented variational formulation and the corresponding Galerkin scheme are well-posed. Then, we obtain a simple a posteriori error estimator and prove that it is reliable and locally efficient. Finally, we provide several numerical experiments that illustrate the theoretical results and support the use of the corresponding adaptive algorithm in practice.
\end{abstract}

Mathematics Subject Classifications (1991): 65N30; 65N12; 65N15

Key words: Darcy flow, mixed finite element, stabilization, a posteriori error estimator.

\section{Introduction}

The problem of Darcy flow is of great importance in civil, geotechnical and petroleum engineering. It describes the flow of a fluid through a porous medium. The natural unknowns are the fluid pressure and the fluid velocity, being the latter the unknown of primary interest in many applications. The problem can be reduced to an elliptic equation for the pressure with a Neumann boundary condition. Although this reduced problem can be solved with appropriate accuracy by a classical Galerkin finite element method, typically there is a loss of accuracy in the approximation of the velocity through the pressure gradients. Moreover, with this reduced formulation mass conservation is not guaranteed. For this reason, the primal formulation for the pressure is not considered adequate for practical engineering applications.

\footnotetext{
*Departamento de Matemática y Física Aplicadas, Universidad Católica de la Santísima Concepción, Casilla 297, Concepción, Chile. E-mail: tomas@ucsc.cl

${ }^{\dagger}$ Departamento de Economía e Historia Económica, Universidad de Salamanca, Salamanca, 37008, Spain. E-mail: casbar@usal.es

${ }^{\ddagger}$ Departamento de Matemáticas, Facultad de Informática, Universidade da Coruña, Campus de Elviña s/n, 15071, A Coruña, Spain. E-mail: maria.gonzalez.taboada@udc.es

${ }_{\S}^{\S}$ Basque Center for Applied Mathematics (BCAM), Alameda de Mazarredo 14, 48009, Bilbao, Spain.
} 
The most popular approach in applications is based on the mixed formulation, with pressure and velocity as unknowns. It is well-known that the Galerkin scheme associated to this formulation is not always well-posed and stability is ensured only for certain combinations of finite element subspaces. In this framework, we consider a stabilized mixed finite element method introduced by Masud and Hughes in [10] for isotropic porous media. This method is based on the addition of suitable residual type terms to the standard dual-mixed approach. The resulting scheme is stable for any combination of continuous velocity and pressure interpolations. Concerning the a posteriori error analysis of this method, a residual based a posteriori error estimate of the velocity in $L^{2}$-norm was derived in [8].

In this paper we consider a generalization of the method proposed in [10] to heterogeneous, possibly anisotropic, porous media flow. We obtained sufficient conditions on the two stabilization parameters such that the augmented dual-mixed variational formulation and the corresponding augmented scheme are well-posed and a Céa-type estimate holds. We remark that these conditions on the stabilization parameters do not depend on the mesh nor on the type of elements employed to solve the discrete problem. Indeed, the parameter related to the mass conservation residual is a non-dimensional, universal constant, that may be selected once and for all. On the other hand, the choice of the parameter related with the residual in Darcy's law depends on the constitutive coefficients of the equation.

Our aim is to develop an a posteriori error analysis of this method. Our analysis is based on the stability of the augmented variational formulation and allows us to derive a simple a posteriori error estimator for the total error. We prove that the new a posteriori error estimator is reliable and locally efficient. We emphasize that these properties hold for any conforming finite element approximation of the continuous problem. Numerical experiments illustrate the confiability and efficiency of the a posteriori error estimator, and support the use of the corresponding adaptive refinement algorithm in practice.

The outline of the paper is as follows. In Section 2 we first describe the problem of Darcy's flow and recall its classical dual-mixed variational formulation. Then, we introduce a slight generalization of the second method analyzed in [10] and provide sufficient conditions on the stabilization parameters that allow to guarantee that the augmented variational formulation is well-posed. In Section 3 we describe the augmented discrete scheme and analyze its stability. We also provide the corresponding rate of convergence for some specific finite element subspaces. The new a posteriori error estimator is derived in Section 4, where we also prove that it is reliable and locally efficient. Finally, some numerical experiments are reported in Section 5. Conclusions are drawn in Section6.

\section{The augmented variational formulation}

We assume that the porous medium $\Omega$ is a bounded connected open domain of $\mathbb{R}^{d}(d=2,3)$ with a Lipschitz-continuous boundary $\Gamma$, and we let $\mathbf{n}$ be the unit outward normal vector to $\Gamma$. We denote by $\mathcal{K} \in\left[L^{\infty}(\Omega)\right]^{d \times d}$ the hydraulic conductivity tensor and assume that it is symmetric and uniformly positive definite, that is, it satisfies

$$
(\mathcal{K}(\mathbf{x}) \mathbf{y}) \cdot \mathbf{y} \geq \alpha\|\mathbf{y}\|^{2}, \quad \text { a.e. } \mathbf{x} \in \Omega, \quad \forall \mathbf{y} \in \mathbb{R}^{d},
$$


for some $\alpha>0$. Then, we also have that $\mathcal{K}^{-1} \in\left[L^{\infty}(\Omega)\right]^{d \times d}$ and

$$
\left(\mathcal{K}^{-1}(\mathbf{x}) \mathbf{y}\right) \cdot \mathbf{y} \geq \frac{\alpha}{\|\mathcal{K}\|^{2}}\|\mathbf{y}\|^{2}, \quad \text { a.e. } \quad \mathbf{x} \in \Omega, \quad \forall \mathbf{y} \in \mathbb{R}^{d} .
$$

We recall that in isotropic porous media, the hydraulic conductivity is a diagonal tensor with diagonal entries equal to the ratio of the permeability, $\kappa>0$, to the viscosity of the fluid, $\mu>0$, that is, $\mathcal{K}=\frac{\kappa}{\mu} \mathbf{I}$, where $\mathbf{I} \in \mathbb{R}^{d \times d}$ is the identity matrix.

In what follows, we denote by $\mathbf{f}:=-\frac{\rho}{g_{c}} \mathbf{g}$, where $\rho>0$ is the fluid density, $\mathbf{g}$ is the gravity acceleration vector and $g_{c}$ is a conversion constant. We let $\varphi$ be the volumetric flow rate source or sink and $\psi$ be the normal component of the velocity field on the boundary. We assume that $\varphi$ and $\psi$ satisfy the compatibility condition $\int_{\Omega} \varphi=\int_{\Gamma} \psi$. Then, the Darcy problem reads: find the fluid velocity $\mathbf{v}: \Omega \rightarrow \mathbb{R}^{d}$ and the pressure $p: \Omega \rightarrow \mathbb{R}$ such that

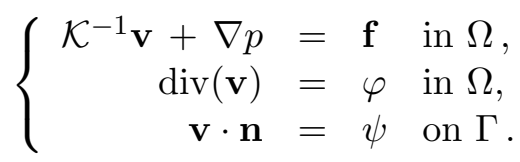

The first equation in (3) is known as Darcy's law and was formulated by H. Darcy in 1856 [4]. The second equation in (3) is the mass conservation equation. This model (3) also appears in other contexts. For instance, it is involved in the projection-diffusion algorithm for solving the timedependent Stokes and Navier-Stokes equations, as suggested by A.J. Chorin [3] and R. Temam [13].

The classical dual-mixed variational formulation of problem (3) reads: find $(\mathbf{v}, p) \in H_{\psi} \times L_{0}^{2}(\Omega)$ such that

$$
\left\{\begin{array}{rlrl}
\int_{\Omega} \mathcal{K}^{-1} \mathbf{v} \cdot \mathbf{w}-\int_{\Omega} p \operatorname{div}(\mathbf{w}) & =\int_{\Omega} \mathbf{f} \cdot \mathbf{w}, & & \forall \mathbf{w} \in H_{0}, \\
\int_{\Omega} \operatorname{div}(\mathbf{v}) q & =\int_{\Omega} \varphi q, & \forall q \in L_{0}^{2}(\Omega),
\end{array}\right.
$$

where for a scalar function $\zeta$, we denote by $H_{\zeta}:=\{\mathbf{w} \in H(\operatorname{div}, \Omega): \mathbf{w} \cdot \mathbf{n}=\zeta$ on $\Gamma\}$ and $L_{0}^{2}(\Omega):=\left\{q \in L^{2}(\Omega): \int_{\Omega} q=0\right\}$. It is well known (see [1]) that, for sufficiently regular data, the weak formulation (4) has a unique solution $(\mathbf{v}, p)$ in $H_{\psi} \times M$, with $M:=H^{1}(\Omega) \cap L_{0}^{2}(\Omega)$.

The dual-mixed variational formulation (4) is the basis of the Galerkin mixed finite element method, for which it is known that only certain combinations of velocity and pressure interpolations are stable. In order to allow a greater set of stable interpolations, we follow [10] and add to the usual dual-mixed variational formulation (4) the following residual type terms, that arise from Darcy's law $(3)_{1}$ and from the mass conservation equation $(3)_{2}$ :

$$
\kappa_{1} \int_{\Omega}\left(\nabla p+\mathcal{K}^{-1} \mathbf{v}\right) \cdot\left(\nabla q-\mathcal{K}^{-1} \mathbf{w}\right)=\kappa_{1} \int_{\Omega} \mathbf{f} \cdot\left(\nabla q-\mathcal{K}^{-1} \mathbf{w}\right), \quad \forall(\mathbf{w}, q) \in H_{0} \times M,
$$

and

$$
\kappa_{2} \int_{\Omega} \operatorname{div}(\mathbf{v}) \operatorname{div}(\mathbf{w})=\kappa_{2} \int_{\Omega} \varphi \operatorname{div}(\mathbf{w}), \quad \forall \mathbf{w} \in H_{0}
$$

where the stabilization parameters $\kappa_{1}$ and $\kappa_{2}$ are positive constants. 
Let $\mathbf{H}:=H(\operatorname{div}, \Omega) \times M$ and let us denote by $\|\cdot\|_{\mathbf{H}}$ the corresponding product norm. By adding equations (4)-(6), we obtain the following stabilized weak variational formulation: find $(\mathbf{v}, p) \in H_{\psi} \times M$ such that

$$
A_{s}((\mathbf{v}, p),(\mathbf{w}, q))=F_{s}(\mathbf{w}, q), \quad \forall(\mathbf{w}, q) \in H_{0} \times M,
$$

where the bilinear form $A_{s}: \mathbf{H} \times \mathbf{H} \rightarrow \mathbb{R}$ and the linear functional $F_{s}: \mathbf{H} \rightarrow \mathbb{R}$ are defined by

$$
\begin{aligned}
& A_{s}((\mathbf{v}, p),(\mathbf{w}, q)):=\int_{\Omega} \mathcal{K}^{-1} \mathbf{v} \cdot \mathbf{w}-\int_{\Omega} p \operatorname{div}(\mathbf{w})+\int_{\Omega} q \operatorname{div}(\mathbf{v}) \\
& +\kappa_{1} \int_{\Omega}\left(\nabla p+\mathcal{K}^{-1} \mathbf{v}\right) \cdot\left(\nabla q-\mathcal{K}^{-1} \mathbf{w}\right)+\kappa_{2} \int_{\Omega} \operatorname{div}(\mathbf{v}) \operatorname{div}(\mathbf{w})
\end{aligned}
$$

and

$$
F_{s}(\mathbf{w}, q):=\int_{\Omega} \mathbf{f} \cdot \mathbf{w}+\int_{\Omega} \varphi q+\kappa_{1} \int_{\Omega} \mathbf{f} \cdot\left(\nabla q-\mathcal{K}^{-1} \mathbf{w}\right)+\kappa_{2} \int_{\Omega} \varphi \operatorname{div}(\mathbf{w}),
$$

for all $(\mathbf{v}, p),(\mathbf{w}, q) \in \mathbf{H}$. At this point, we remark that the stabilization methods analyzed in [10] for isotropic porous media correspond to the choices $\kappa_{1}=\frac{\kappa}{2 \mu}, \kappa_{2}=0$ and $\kappa_{1}=\frac{\kappa}{2 \mu}, \kappa_{2}=\frac{c}{2} \frac{\mu}{\kappa} h^{2}$, where $c=\mathcal{O}(1)$ and $h$ is the mesh size, assuming $\kappa$ and $\mu$ are constant.

Lemma 2.1 Assume that $\kappa_{1} \in\left(0, \frac{\alpha}{\|\mathcal{K}\|^{2}\left\|\mathcal{K}^{-1}\right\|^{2}}\right)$ and $\kappa_{2}>0$. Then, the bilinear form $A_{s}(\cdot, \cdot)$ is elliptic in $\mathbf{H}$, that is, there exists $C_{\mathrm{e} 11}>0$ such that

$$
A_{s}((\mathbf{w}, q),(\mathbf{w}, q)) \geq C_{\mathrm{ell}}\|(\mathbf{w}, q)\|_{\mathbf{H}}^{2}, \quad \forall(\mathbf{w}, q) \in \mathbf{H} .
$$

Proof. Let $(\mathbf{w}, q) \in \mathbf{H}$. Then, using the definition of $A_{s}(\cdot, \cdot),(2)$ and that $\mathcal{K}^{-1} \in\left[L^{\infty}(\Omega)\right]^{d \times d}$, we obtain

$$
\begin{aligned}
A_{s}((\mathbf{w}, q),(\mathbf{w}, q)) & =\int_{\Omega} \mathcal{K}^{-1} \mathbf{w} \cdot \mathbf{w}+\kappa_{1} \int_{\Omega}|\nabla q|^{2}-\kappa_{1} \int_{\Omega}\left|\mathcal{K}^{-1} \mathbf{w}\right|^{2}+\kappa_{2} \int_{\Omega}|\operatorname{div}(\mathbf{w})|^{2} \\
& \geq\left(\frac{\alpha}{\|\mathcal{K}\|^{2}}-\kappa_{1}\left\|\mathcal{K}^{-1}\right\|^{2}\right)\|\mathbf{w}\|_{\left[L^{2}(\Omega)\right]^{d}}^{2}+\kappa_{1}\|\nabla q\|_{\left[L^{2}(\Omega)\right]^{d}}^{2}+\kappa_{2}\|\operatorname{div}(\mathbf{w})\|_{L^{2}(\Omega)}^{2}
\end{aligned}
$$

The proof follows by applying the Poincaré inequality in $L_{0}^{2}(\Omega)$.

Theorem 2.1 Under the hypotheses of Lemma 2.1 problem (7) has a unique solution $(\mathbf{v}, p) \in$ $H_{\psi} \times M$.

Proof. The result follows from the previous Lemma and the Lax-Milgram Lemma.

We remark that, under the assumptions of Lemma 2.1, the ellipticity constant $C_{\text {ell }}$ can be chosen as

$$
C_{\text {ell }}:=\min \left(\frac{\alpha}{\|\mathcal{K}\|^{2}}-\kappa_{1}\left\|\mathcal{K}^{-1}\right\|^{2}, \frac{\kappa_{1}}{2} \min \left(1, C_{\Omega}^{2}\right), \kappa_{2}\right),
$$

where $C_{\Omega}$ is the Poincaré constant. We also observe that for the case considered in $[10]\left(\mathcal{K}=\frac{\kappa}{\mu} \mathbf{I}\right.$, with $\kappa$ and $\mu$ positive constants), it suffices to take $\kappa_{1} \in\left(0, \frac{\kappa}{\mu}\right)$ and $\kappa_{2}>0$. This explains the good behavior of the second method introduced in [10], but not that of the first one. With the previous analysis, we cannot ensure the well-posedness of the augmented variational formulation (7) when $\kappa_{2}=0$. 


\section{The augmented mixed finite element method}

In what follows, we assume that the stabilization parameters $\kappa_{1}$ and $\kappa_{2}$ satisfy the hypothesis of Lemma 2.1 and Theorem 2.1. We also assume that $\Omega$ is a polygonal or polyhedral domain.

Let $H_{h}$ and $M_{h}$ be any finite dimensional subspaces of $H(\operatorname{div}, \Omega)$ and $M$, respectively. We denote by $H_{\zeta, h}:=H_{\zeta} \cap H_{h}$. Then, the Galerkin scheme associated to problem (7) reads: find $\left(\mathbf{v}_{h}, p_{h}\right) \in H_{\psi, h} \times M_{h}$ such that

$$
A_{s}\left(\left(\mathbf{v}_{h}, p_{h}\right),\left(\mathbf{w}_{h}, q_{h}\right)\right)=F_{s}\left(\mathbf{w}_{h}, q_{h}\right), \quad \forall\left(\mathbf{w}_{h}, q_{h}\right) \in H_{0, h} \times M_{h} .
$$

Thanks to the ellipticity of the bilinear form $A_{s}(\cdot, \cdot)$ in $\mathbf{H}$, problem (9) has a unique solution $\left(\mathbf{v}_{h}, p_{h}\right) \in H_{\psi, h} \times M_{h}$. Moreover, there exists a constant $C>0$, independent of $h$, such that

$$
\left\|\left(\mathbf{v}-\mathbf{v}_{h}, p-p_{h}\right)\right\|_{\mathbf{H}} \leq C \inf _{\left(\mathbf{w}_{h}, q_{h}\right) \in H_{0, h} \times M_{h}}\left\|\left(\mathbf{v}-\mathbf{w}_{h}, p-q_{h}\right)\right\|_{\mathbf{H}} .
$$

In order to establish a rate of convergence result, we consider specific finite element subspaces $H_{h}$ and $M_{h}$. Let $\left\{\mathcal{T}_{h}\right\}_{h>0}$ be a family of shape-regular meshes of $\bar{\Omega}$ made up of triangles in $2 \mathrm{D}$ or tetrahedra in 3D. We denote by $h_{T}$ the diameter of an element $T \in \mathcal{T}_{h}$ and define $h:=\max _{T \in \mathcal{T}_{h}} h_{T}$. Hereafter, given $T \in \mathcal{T}_{h}$ and an integer $l \geq 0$, we denote by $\mathcal{P}_{l}(T)$ the space of polynomials of total degree at most $l$ on $T$. Now, let $H_{h} \subset H(\operatorname{div} ; \Omega)$ be either the Raviart-Thomas space of order $r \geq 0$ (cf. [11]), i.e.

$$
H_{h}=\mathcal{R} \mathcal{T}_{r}\left(\mathcal{T}_{h}\right):=\left\{\mathbf{w}_{h} \in H(\operatorname{div} ; \Omega): \mathbf{w}_{h \mid T} \in\left(\left[\mathcal{P}_{r}(T)\right]^{d}+\mathbf{x} \mathcal{P}_{r}(T)\right), \quad \forall T \in \mathcal{T}_{h}\right\}
$$

where $\mathbf{x} \in \mathbb{R}^{d}$ is a generic vector, or the Brezzi-Douglas-Marini space of order $r+1, r \geq 0$ (cf. [1]), i.e.

$$
H_{h}=\mathcal{B D} \mathcal{M}_{r+1}\left(\mathcal{T}_{h}\right):=\left\{\mathbf{w}_{h} \in H(\operatorname{div} ; \Omega): \mathbf{w}_{h \mid T} \in\left[\mathcal{P}_{r+1}(T)\right]^{d}, \quad \forall T \in \mathcal{T}_{h}\right\} .
$$

In Figure 1 we show the degrees of freedom for the Raviart-Thomas elements of order 0 and 1, and for the Brezzi-Douglas-Marini element of order 1.
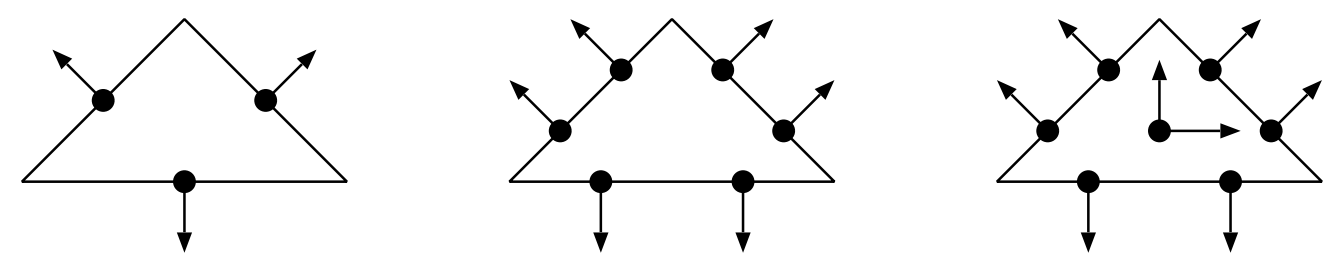

Figure 1: Degrees of freedom for $\mathcal{R} \mathcal{T}_{0}$ (left), $\mathcal{B D} \mathcal{M}_{1}$ (center) and $\mathcal{R} \mathcal{T}_{1}$ (right) on a triangle $T$.

We also introduce the standard Lagrange space of order $m \geq 1$ :

$$
M_{h}:=\mathcal{L}_{m}\left(\mathcal{T}_{h}\right)=\left\{q_{h} \in \mathcal{C}(\bar{\Omega}) \cap L_{0}^{2}(\Omega):\left.q_{h}\right|_{T} \in \mathcal{P}_{m}(T), \quad \forall T \in \mathcal{T}_{h}\right\}
$$

The corresponding a priori error bound is given in the next theorem. 
Theorem 3.1 Assume $0<\kappa_{1}<\frac{\alpha}{\|\mathcal{K}\|^{2}\left\|\mathcal{K}^{-1}\right\|^{2}}$ and $\kappa_{2}>0$. Then, if $\mathbf{v} \in\left[H^{t}(\Omega)\right]^{d}, \operatorname{div}(\mathbf{v}) \in H^{t}(\Omega)$ and $p \in H^{t+1}(\Omega)$, there exists $C>0$, independent of $h$, such that

$$
\left\|\left(\mathbf{v}-\mathbf{v}_{h}, p-p_{h}\right)\right\|_{\mathbf{H}} \leq C h^{\min \{t, m, r+1\}}\left(\|\mathbf{v}\|_{\left[H^{t}(\Omega)\right]^{d}}+\|\operatorname{div}(\mathbf{v})\|_{H^{t}(\Omega)}+\|p\|_{H^{t+1}(\Omega)}\right) .
$$

Proof. It follows straightforwardly from the Céa estimate (10) and the approximation properties of the corresponding finite element subspaces (cf. [1]).

\section{A posteriori error analysis}

In this section, we develop a residual-based a posteriori error analysis of the augmented discrete scheme (9). We derive a simple a posteriori error estimator that requires the computation of two residuals per element and we show that it is reliable and locally efficient.

Let $H_{h} \times M_{h}$ be any finite element subspace of $H(\operatorname{div}, \Omega) \times M$. Throughout this section, we assume that $0<\kappa_{1}<\frac{\alpha}{\|\mathcal{K}\|^{2}\left\|\mathcal{K}^{-1}\right\|^{2}}, \kappa_{2}>0$ and we let $(\mathbf{v}, p) \in H_{\psi} \times M$ and $\left(\mathbf{v}_{h}, p_{h}\right) \in H_{\psi, h} \times M_{h}$ be the unique solutions to problems (7) and (9), respectively. Then, we consider the residual

$$
R_{h}(\mathbf{w}, q):=F_{s}(\mathbf{w}, q)-A_{s}\left(\left(\mathbf{v}_{h}, p_{h}\right),(\mathbf{w}, q)\right), \quad \forall(\mathbf{w}, q) \in H_{0} \times M .
$$

Using the ellipticity of the bilinear form $A_{s}(\cdot, \cdot)$ and the definition of the residual (11), we have

$$
\begin{aligned}
\left\|\left(\mathbf{v}-\mathbf{v}_{h}, p-p_{h}\right)\right\|_{\mathbf{H}} & \leq C_{\mathrm{ell}}^{-1} \frac{A_{s}\left(\left(\mathbf{v}-\mathbf{v}_{h}, p-p_{h}\right),\left(\mathbf{v}-\mathbf{v}_{h}, p-p_{h}\right)\right)}{\left\|\left(\mathbf{v}-\mathbf{v}_{h}, p-p_{h}\right)\right\|_{\mathbf{H}}} \\
& \leq C_{\mathrm{ell}}^{-1} \sup _{\substack{(\mathbf{w}, q) \in H_{0} \times M \\
(\mathbf{w}, q) \neq(0,0)}} \frac{A_{s}\left(\left(\mathbf{v}-\mathbf{v}_{h}, p-p_{h}\right),(\mathbf{w}, q)\right)}{\|(\mathbf{w}, q)\|_{\mathbf{H}}} \\
& =C_{\mathrm{ell}}^{-1} \sup _{\substack{(\mathbf{w}, q) \in H_{0} \times M \\
(\mathbf{w}, q) \neq(0,0)}} \frac{R_{h}(\mathbf{w}, q)}{\|(\mathbf{w}, q)\|_{\mathbf{H}}} .
\end{aligned}
$$

In the next Lemma, we obtain an upper bound for the residual.

Lemma 4.1 There exists a positive constant $C$, independent of $h$, such that

$$
\sup _{\substack{(\mathbf{w}, q) \in H_{0} \times M \\(\mathbf{w}, q) \neq(0,0)}} \frac{R_{h}(\mathbf{w}, q)}{\|(\mathbf{w}, q)\|_{\mathbf{H}}} \leq C\left(\left\|\mathbf{f}-\nabla p_{h}-\mathcal{K}^{-1} \mathbf{v}_{h}\right\|_{\left[L^{2}(\Omega)\right]^{d}}+\left\|\varphi-\operatorname{div}\left(\mathbf{v}_{h}\right)\right\|_{L^{2}(\Omega)}\right) .
$$

Proof. Let $(\mathbf{w}, q) \in H_{0} \times M$. Using the definitions of the linear functional $F_{s}$ and the bilinear form $A_{s}(\cdot, \cdot)$, we can write

$$
R_{h}(\mathbf{w}, q)=R_{1}(\mathbf{w})+R_{2}(q), \quad \forall \mathbf{w} \in H_{0}, \quad \forall q \in M,
$$


where

$$
\begin{gathered}
R_{1}(\mathbf{w}):=\int_{\Omega} \mathbf{f} \cdot \mathbf{w}+\int_{\Omega} p_{h} \operatorname{div}(\mathbf{w})-\int_{\Omega} \mathcal{K}^{-1} \mathbf{v}_{h} \cdot \mathbf{w}-\kappa_{1} \int_{\Omega}\left(\mathbf{f}-\nabla p_{h}-\mathcal{K}^{-1} \mathbf{v}_{h}\right) \cdot \mathcal{K}^{-1} \mathbf{w} \\
+\kappa_{2} \int_{\Omega}\left(\varphi-\operatorname{div}\left(\mathbf{v}_{h}\right)\right) \operatorname{div}(\mathbf{w})
\end{gathered}
$$

and

$$
R_{2}(q):=\int_{\Omega}\left(\varphi-\operatorname{div}\left(\mathbf{v}_{h}\right)\right) q+\kappa_{1} \int_{\Omega}\left(\mathbf{f}-\nabla p_{h}-\mathcal{K}^{-1} \mathbf{v}_{h}\right) \cdot \nabla q .
$$

Then, integrating by parts the second term on the right hand side of (14), using that $\mathbf{w} \cdot \mathbf{n}=0$ on $\Gamma$ and applying the Cauchy-Schwarz inequality and the continuity of $\mathcal{K}^{-1}$, we have

$$
\left|R_{1}(\mathbf{w})\right| \leq\left(\left(1+\kappa_{1}\left\|\mathcal{K}^{-1}\right\|\right)\left\|\mathbf{f}-\nabla p_{h}-\mathcal{K}^{-1} \mathbf{v}_{h}\right\|_{\left[L^{2}(\Omega)\right]^{d}}+\kappa_{2}\left\|\varphi-\operatorname{div}\left(\mathbf{v}_{h}\right)\right\|_{L^{2}(\Omega)}\right)\|\mathbf{w}\|_{H(\operatorname{div}, \Omega)} .
$$

On the other hand, the Cauchy-Schwarz inequality also implies that

$$
\left|R_{2}(q)\right| \leq\left(\kappa_{1}\left\|\mathbf{f}-\nabla p_{h}-\mathcal{K}^{-1} \mathbf{v}_{h}\right\|_{\left[L^{2}(\Omega)\right]^{d}}+\left\|\varphi-\operatorname{div}\left(\mathbf{v}_{h}\right)\right\|_{L^{2}(\Omega)}\right)\|q\|_{H^{1}(\Omega)} .
$$

Then, the proof follows by applying the triangle inequality in (13) and using (15) and (16). We remark that $C$ can be chosen as

$$
C:=\max \left(1+\kappa_{1}\left(1+\left\|\mathcal{K}^{-1}\right\|\right), 1+\kappa_{2}\right) .
$$

Motivated by inequality (12) and the previous result, we define the a posteriori error estimator $\eta_{h}$ as follows:

$$
\eta_{h}^{2}:=\sum_{T \in \mathcal{T}_{h}} \eta_{h}^{2}(T), \quad \text { with } \quad \eta_{h}^{2}(T):=\left\|\mathbf{f}-\nabla p_{h}-\mathcal{K}^{-1} \mathbf{v}_{h}\right\|_{\left[L^{2}(T)\right]^{d}}^{2}+\left\|\varphi-\operatorname{div}\left(\mathbf{v}_{h}\right)\right\|_{L^{2}(T)}^{2} .
$$

We remark that the local error indicator $\eta_{h}(T)$ consists of two residual terms, namely, the local residual in Darcy's law and the local residual in the mass conservation equation. We also notice that the global a posteriori error estimator $\eta_{h}$ does not involve the computation of any jump across the elements of the mesh. This fact, besides the good properties of the estimator stated in the next Theorem, make $\eta_{h}$ well-suited for numerical computations.

Theorem 4.1 There exists a positive constant $C_{\mathrm{rel}}$, independent of $h$, such that

$$
\left\|\left(\mathbf{v}-\mathbf{v}_{h}, p-p_{h}\right)\right\|_{\mathbf{H}} \leq C_{\mathrm{rel}} \eta_{h},
$$

and there exists a positive constant $C_{\mathrm{eff}}$, independent of $h$ and $T$, such that

$$
C_{\text {eff }} \eta_{h}(T) \leq\left\|\left(\mathbf{v}-\mathbf{v}_{h}, p-p_{h}\right)\right\|_{H(d i v, T) \times H^{1}(T)}, \quad \forall T \in \mathcal{T}_{h} .
$$


Proof. The first inequality is a consequence of (12), Lemma 4.1 and the definition of $\eta_{h}$. In fact, we can take $C_{\mathrm{rel}}:=\sqrt{2} C / C_{\mathrm{e} 11}$, where $C_{\mathrm{ell}}$ and $C$ are the constants defined in (8) and (17), respectively. On the other hand, we recall that $\operatorname{div}(\mathbf{v})=\varphi$ and $\mathbf{f}=\nabla p+\mathcal{K}^{-1} \mathbf{v}$ in $\Omega$. Then, we have that

$$
\left\|\varphi-\operatorname{div}\left(\mathbf{v}_{h}\right)\right\|_{L^{2}(T)}=\left\|\operatorname{div}\left(\mathbf{v}-\mathbf{v}_{h}\right)\right\|_{L^{2}(T)}
$$

and, using the triangle inequality and the continuity of $\mathcal{K}^{-1}$,

$$
\left\|\mathbf{f}-\nabla p_{h}-\mathcal{K}^{-1} \mathbf{v}_{h}\right\|_{\left[L^{2}(T)\right]^{d}} \leq \max \left\{\left\|\mathcal{K}^{-1}\right\|, 1\right\}\left(\left\|\mathbf{v}-\mathbf{v}_{h}\right\|_{\left[L^{2}(T)\right]^{d}}+\left\|\nabla\left(p-p_{h}\right)\right\|_{\left[L^{2}(T)\right]^{d}}\right) .
$$

Then, (19) follows with $C_{\text {eff }}^{-1}:=\sqrt{3} \max \left(1,\left\|\mathcal{K}^{-1}\right\|\right)$.

Theorem 4.1 establishes the equivalence between the total error and the estimator $\eta_{h}$. Inequality (18) means that the a posteriori error estimator $\eta_{h}$ is reliable, whereas inequality (19) means that $\eta_{h}$ is locally efficient. We remark that the efficiency constant $C_{\text {eff }}$ can be chosen independently of the stabilization parameters.

\section{$5 \quad$ Numerical results}

In this section we present some numerical experiments that illustrate the confiability and efficiency of the a posteriori error estimator $\eta_{h}$. For implementation purposes, instead of imposing the null media condition required to the elements of $M_{h}$, we fix the value of the pressure on a point of the numerical domain. The experiments have been performed with the finite element toolbox ALBERTA (cf. [12]) using refinement by recursive bisection [7]. The solution of the corresponding linear systems has been computed using the SuperLU library [5]. We present numerical experiments for the finite element pairs $\left(H_{h}, M_{h}\right)$ given by $\left(\mathcal{R} \mathcal{T}_{0}, \mathcal{L}_{1}\right),\left(\mathcal{R} \mathcal{T}_{1}, \mathcal{L}_{2}\right)$ and $\left(\mathcal{B D} \mathcal{M}_{1}, \mathcal{L}_{1}\right)$ in the twodimensional case and $\left(\mathcal{R} \mathcal{T}_{0}, \mathcal{L}_{1}\right)$ in three dimensions.

We use the standard adaptive finite element method (AFEM) based on the loop:

$$
\mathrm{SOLVE} \rightarrow \text { ESTIMATE } \rightarrow \text { MARK } \rightarrow \text { REFINE. }
$$

Hereafter, we replace the subscript $h$ by $k$, where $k$ is the counter of the adaptive loop. Then, given a mesh $\mathcal{T}_{k}$, the procedure SOLVE is an efficient direct solver for computing the discrete solution $\left(\mathbf{v}_{k}, p_{k}\right)$, ESTIMATE calculates the error indicators $\eta_{k}(T)$, for all $T \in \mathcal{T}_{k}$, depending on the computed solution and the data. Based on the values of $\left\{\eta_{k}(T)\right\}_{T \in \mathcal{T}_{k}}$, the procedure MARK generates a set of marked elements subject to refinement. For the elements selection, we rely on the maximum strategy: given a threshold $\sigma \in(0,1]$, elements $T \in \mathcal{T}_{k}$ such that

$$
\eta_{k}(T)>\sigma \max _{T \in \mathcal{T}_{k}} \eta_{k}(T)
$$

are marked for refinement. In our experiments, we fix $\sigma=0.6$. Finally, the procedure REFINE creates a conforming refinement $\mathcal{T}_{k+1}$ of $\mathcal{T}_{k}$, bisecting $d$ times all marked elements (where $d=2,3$ is the space dimension). 
In what follows, we present three numerical experiments. The first one is devoted to study the robustness of the method with respect to the stabilization parameters, $\kappa_{1}$ and $\kappa_{2}$, and the rate of convergence when the solution is smooth. In the two subsequent examples, we compare the performance of a finite element method based on uniform refinement (FEM) with the adaptive algorithm described above (AFEM). In Section 5.2, we study the performance of the AFEM algorithm when there are abrupt changes in the hydraulic conductivity tensor and the solution is not smooth. In Section 5.3 we consider a tracer injection-type simulation in three dimensions with a smooth solution. In all the examples, gravity effects are neglected, as it is often done in applications.

\subsection{Example 1: Robustness and convergence rates}

In order to study the robustness of the augmented scheme (9) with respect to the stabilization parameters and the sensitivity of the stabilized formulation to the ratio of the permeability to the viscosity, we consider an example with a smooth solution. We let $\Omega=(0,1)^{2}$ be the unit square, $\mathcal{K}=\frac{\kappa}{\mu} \mathbf{I}, p(x, y)=\sin (2 \pi x) \sin (2 \pi y), \mathbf{f}=\mathbf{0}, \mathbf{v}=-\mathcal{K} \nabla p, \varphi=\operatorname{div}(\mathbf{v})$ and $\psi=\mathbf{v} \cdot \mathbf{n}$.
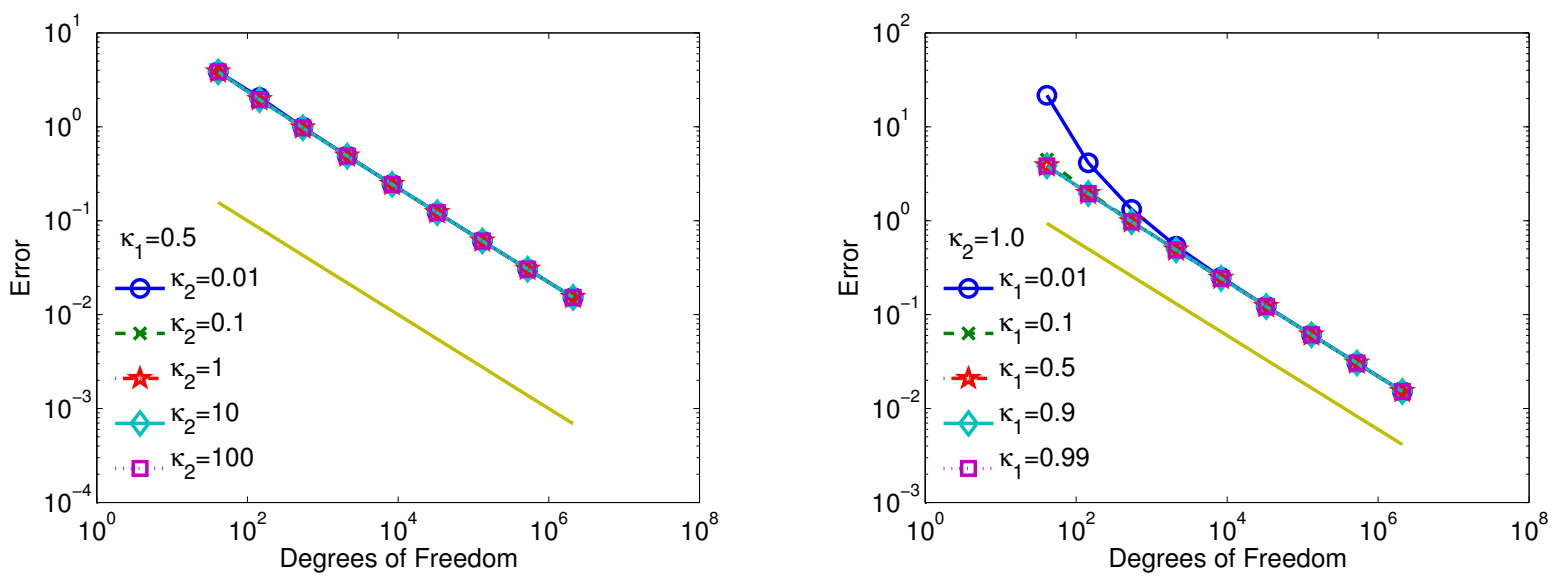

Figure 2: Example 1. Decay of total error for $\kappa / \mu=1$ and several values of $\kappa_{1}$ and $\kappa_{2}$.

We first consider $\mathcal{K}=\mathbf{I}$ and investigate the effect of the stabilization parameters $\kappa_{1}$ and $\kappa_{2}$ on the augmented scheme (9) when the finite element pair $\left(\mathcal{R} \mathcal{T}_{0}, \mathcal{L}_{1}\right)$ is used. Figure 2 shows the decay of the total error versus the degrees of freedom (DOFs) for several values of $\kappa_{1}$ and $\kappa_{2}$, all of them satisfying the hypotheses of Lemma 2.1. Optimal rates are attained in all cases, showing the robustness of the method with respect to the stabilization parameters.

According to these results, in what follows, we choose $\kappa_{1}=\frac{\kappa}{2 \mu}$ and $\kappa_{2}=1.0$. We remark that these values of the stabilization parameters are consistent with the theory and ensure that the bilinear form $A_{s}(\cdot, \cdot)$ is elliptic in the whole space. Then, we solve problem (9) using the finite element pairs $\left(\mathcal{R} \mathcal{T}_{0}, \mathcal{L}_{1}\right),\left(\mathcal{R} \mathcal{T}_{1}, \mathcal{L}_{2}\right)$ and $\left(\mathcal{B D} \mathcal{M}_{1}, \mathcal{L}_{1}\right)$ on a sequence of uniform meshes (i.e., in each step all elements of the actual mesh are bisected twice). Figures 3, 4, 5 and 6 show the decay 
of the total error and the a posteriori error estimator versus the number of DOFs for $\frac{\kappa}{\mu}=10^{-i}$, $i=0, \ldots, 3$, respectively. We observe that the theoretical convergence rates predicted by the theory are achieved in all cases (we recall that $h \sim \mathrm{DOFs}^{-1 / d}$ on uniform meshes). Moreover, the estimator shows the same decay as the error.
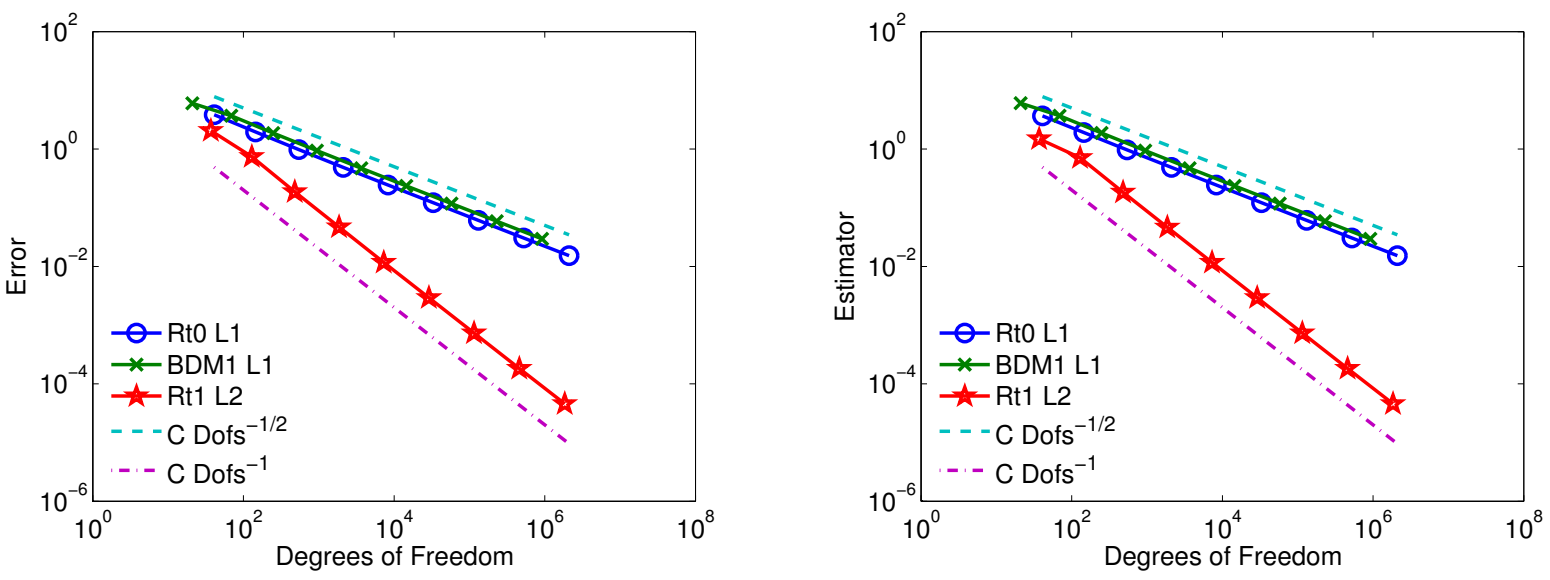

Figure 3: Example 1. Decays of total error (left) and estimator (right) vs. DOFs for $\kappa / \mu=1$.
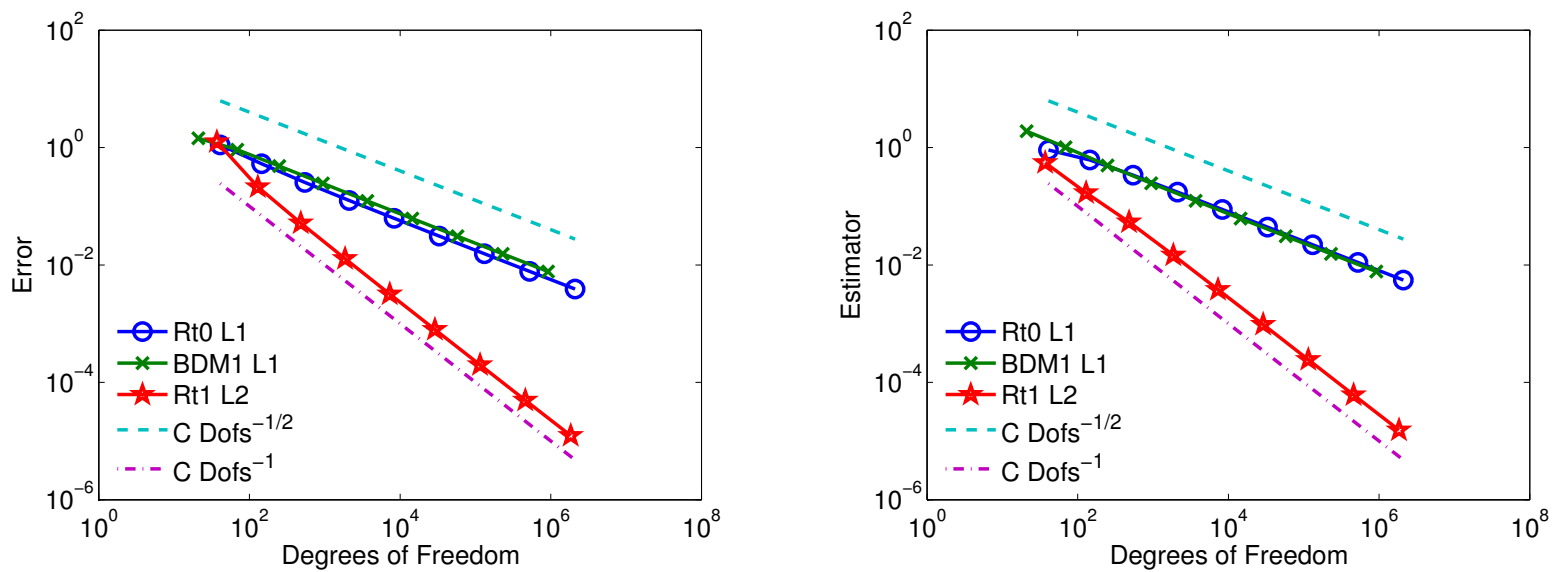

Figure 4: Example 1. Decays of total error (left) and estimator (right) vs. DOFs for $\kappa / \mu=0.1$.

Finally, Figure 7 shows the efficiency indices (defined as the ratio of the estimated error to the total error) for the three finite element pairs considered here. As predicted by the theory, the efficiency index is bounded from below and above by positive constants, independently of the mesh size. In particular, when $\kappa=\mu$, they approach one for the three discretization methods. We also remark that the efficiency indices obtained with the $\mathcal{B D} \mathcal{M}_{1}$-discretization approach one in all cases. This can be explained by the fact that the decay for the $L_{2}$-norm of the velocity with the $\mathcal{B D}_{1}$ 

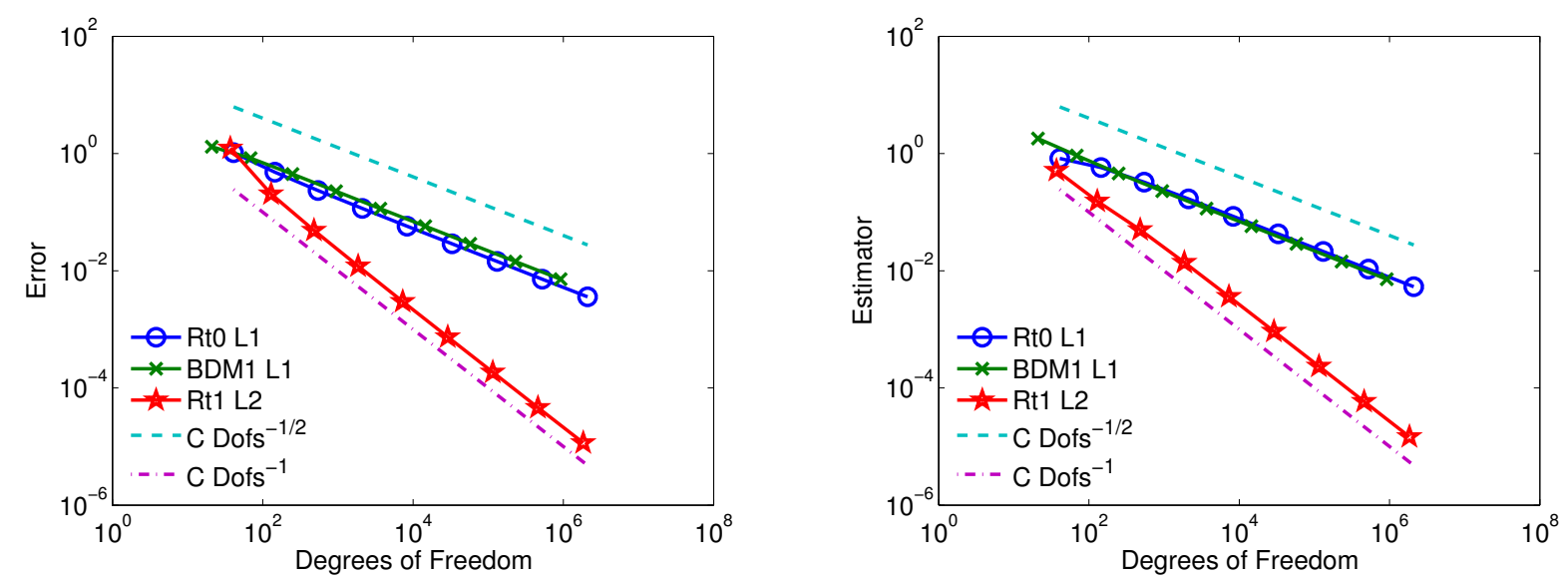

Figure 5: Example 1. Decays of total error (left) and estimator (right) vs. DOFs for $\kappa / \mu=0.01$.
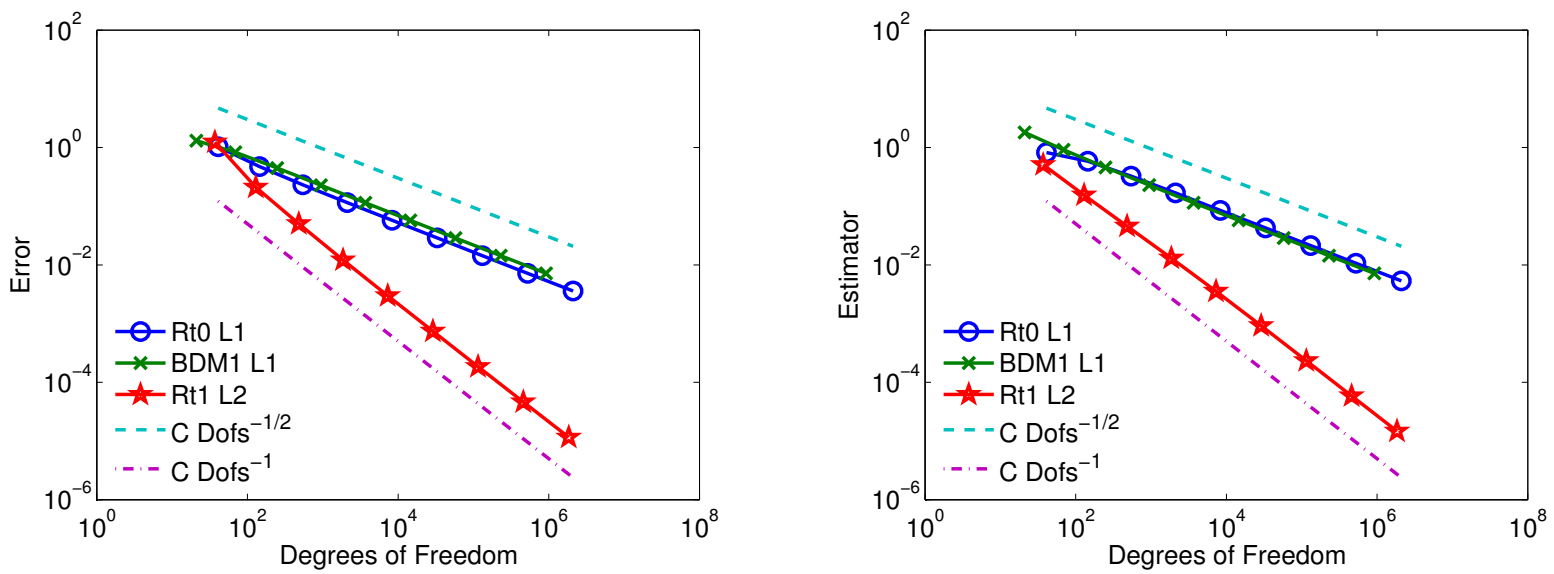

Figure 6: Example 1. Decays of total error (left) and estimator (right) vs. DOFs for $\kappa / \mu=0.001$.

method $\left(h^{2} \sim \mathrm{DOFs}^{-2 / d}\right)$ is higher than for the $H($ div $)$-norm of the velocity $\left(h^{1} \sim \mathrm{DOFs}^{-1 / d}\right)$. Since the contribution of the $L_{2}$-norm of the error in the velocity in the total error is negligible for small $h$ in $\mathcal{B D} \mathcal{M}_{1}$, the estimator seems to be asymptotically exact in this case.

\subsection{Example 2: A checkerboard configuration}

We use this example, taken from [2], to simulate the situation in which there are abrupt changes in the permeability. Let $\Omega=(-1,1)^{2}$ and let $\mathcal{K}=\mathcal{K}_{\gamma}$ be piecewise constant in sectors with a vertex at the origin. More precisely, we let $\mathcal{K}_{\gamma}=a_{1}(\gamma) \mathbf{I}$ in the first and third quadrants, and $\mathcal{K}_{\gamma}=a_{2}(\gamma) \mathbf{I}$ in the second and fourth quadrants. We consider Kellogg's exact solution to the elliptic equation 

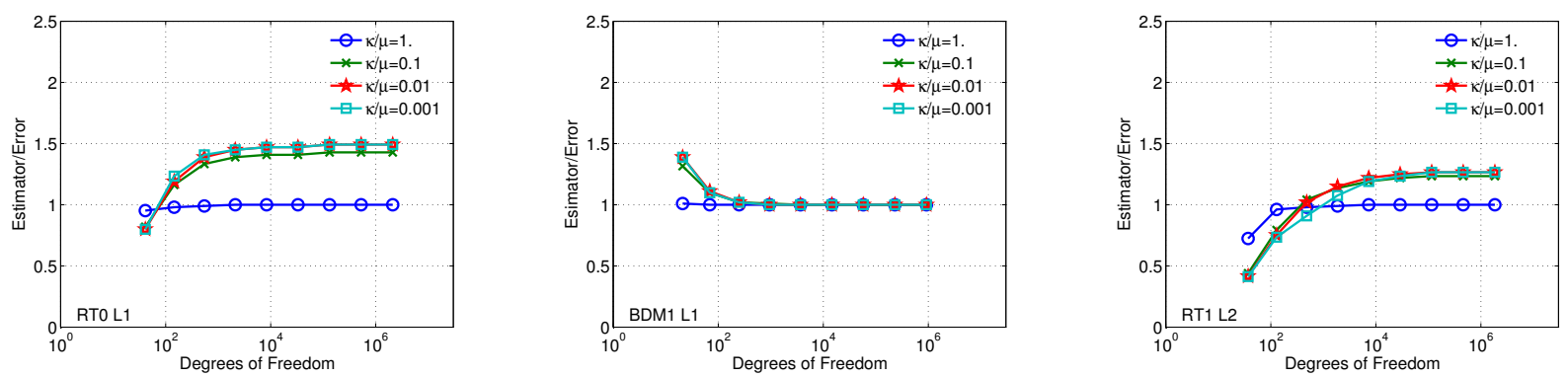

Figure 7: Example 1. Efficiency indices for $\left(\mathcal{R} \mathcal{T}_{0}, \mathcal{L}_{1}\right)$ (left), $\left(\mathcal{B D} \mathcal{M}_{1}, \mathcal{L}_{1}\right)$ (center) and $\left(\mathcal{R} \mathcal{T}_{1}, \mathcal{L}_{2}\right)$ (right).

(cf. [6])

$$
-\operatorname{div}\left(\mathcal{K}_{\gamma} \nabla p\right)=0 \quad \text { in } \Omega
$$

with appropriate Dirichlet boundary conditions for two different values of $\gamma$, namely, $\gamma=0.50$ and $\gamma=0.25$. For these values of the parameter $\gamma$, we have that

$$
a_{1}(0.50)=a_{1}(0.25)=1, \quad a_{2}(\gamma) \cong \begin{cases}0.171572875253810, & \text { if } \gamma=0.50 \\ 0.039566129896580, & \text { if } \gamma=0.25\end{cases}
$$

Kellogg showed (cf. [6]) that an exact solution of (21) is given in polar coordinates by $p(r, \theta)=$ $r^{\gamma} m(\theta)$, where $m$ is a smooth function that depends on $\theta$. We recall that $p$ has the regularity $H^{1+\gamma-\epsilon}$. Then, $\left(\mathbf{v}_{\gamma}, p_{\gamma}\right)$, with $\mathbf{v}_{\gamma}=-\mathcal{K}_{\gamma} \nabla p_{\gamma}$, is the exact solution of problem (3) when $\mathcal{K}=\mathcal{K}_{\gamma}$, $\mathbf{f}=\mathbf{0}, \varphi=0$ and $\psi=\mathbf{v}_{\gamma} \cdot \mathbf{n}$.

We remark that the checkerboard pattern is the most demanding configuration in terms of regularity. We solve both problems with the finite element pair $\left(\mathcal{R} \mathcal{T}_{0}, \mathcal{L}_{1}\right)$ using uniform refinement (FEM algorithm) and the adaptive refinement algorithm (AFEM) described at the beginning of this section. We choose $\kappa_{1}(\gamma)=\frac{a_{2}(\gamma)^{3}}{2}$ and $\kappa_{2}=1.0$, and fix to zero the value of the pressure in a corner of the domain.

Figure 8 shows the decay of the total error and the a posteriori error estimator for the uniform (FEM) and adaptive (AFEM) refinements and the two values of $\gamma$. We remark that the usual FEM algorithm is suboptimal in this case due to the poor regularity of the solution. In turn, the AFEM appears to be quasi-optimal. Efficiency indices are reported in Figure 9. There, we observe that they are bounded from above and below, which confirms that error and estimator are equivalent.

The adapted meshes obtained with this algorithm are highly graded at the origin, as can be observed in Figures 10 and 11 for $\gamma=0.50$, and specially in Figures 12 and 13 for $\gamma=0.25$.

Finally, in Figure 14 we present the pressure obtained after 20 AFEM iterations for $\gamma=0.5$ (24128 triangles) and $\gamma=0.25$ (1744 triangles). In Figure 15 we show the velocity fields obtained after 16 AFEM-iterations for $\gamma=0.50$ (6228 triangles) and for $\gamma=0.25$ (968 triangles). We observe that there are no oscillation in the pressure for the two values of $\gamma$ considered here, which confirms the stability of the method. We can also observe that the velocity is higher in the regions with greater values of the permeability. 

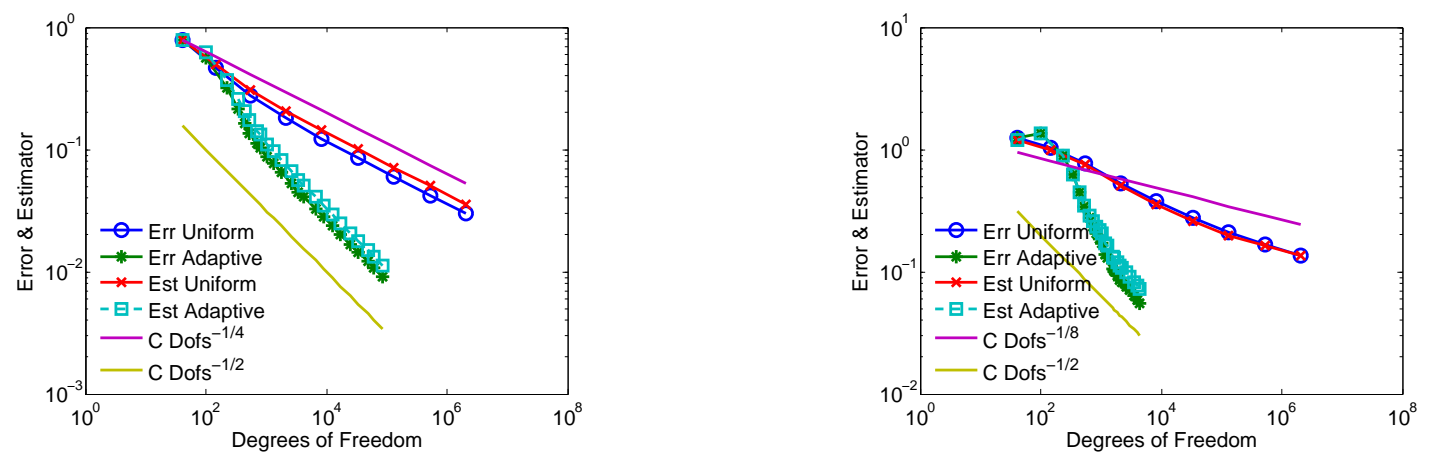

Figure 8: Example 2. Decays of total error and estimator for $\left(\mathcal{R} \mathcal{T}_{0}, \mathcal{L}_{1}\right)$ with FEM and AFEM, for $\gamma=0.50$ (left) and $\gamma=0.25$ (right).

\subsection{Example 3: Tracer injection}

This last example illustrates the performance of our algorithm on a tracer injection type problem with a smooth solution. Let $\Omega=(0,1)^{3}$ be the unit cube. Motivated by Example 5.1 in [9], we choose the data of problem (3) so that the exact solution is $(\mathbf{v}, p)$, with

$$
p(x, y, z)=\log \left(\tan ^{2}\left(L_{\epsilon} r_{\epsilon}(x, y, z)\right)\right),
$$

where $\epsilon$ is a positive number, $r_{\epsilon}(x, y, z)=\sqrt{(x+\epsilon)^{2}+(y+\epsilon)^{2}+(z+\epsilon)^{2}}, L_{\epsilon}=\frac{\pi}{2 \sqrt{3}(1+2 \epsilon)}$, and $\mathbf{v}=-\nabla p$ (that is, $\mathcal{K}=\mathbf{I}$ ). We remark that there is a point sink at $x=y=z=-\epsilon$ and a point source at $x=y=z=1+\epsilon$. Since $\epsilon>0$, the singularities are located outside the computational domain.

We recall that the tracer injection problem can be modeled using a Dirac delta (as in [10]); however, we are interested in comparing the exact error with the a posteriori error estimator $\eta_{k}$. In order to simulate the situation where source and sink are close to the boundary, we choose $\epsilon=10^{-2}$. The stabilization parameters are fixed to $\kappa_{1}=\frac{1}{2}$ and $\kappa_{2}=1.0$. We solve the problem for the finite element pair $\left(\mathcal{R} \mathcal{T}_{0}, \mathcal{L}_{1}\right)$ using the uniform refinement $(\mathrm{FEM})$ and the adaptive refinement (AFEM) algorithms. Although the solution $(\mathbf{v}, p)$ is smooth in $\Omega$, the uniform refinement procedure presents a long plate in the pre-asymptotic regime (see Figure 16). On the other hand, the adaptive refinement algorithm is able to attain linear convergence, revealing itself as a very competitive algorithm. In this case (Figure 16, right), the estimator seems to be asymptotically exact. 

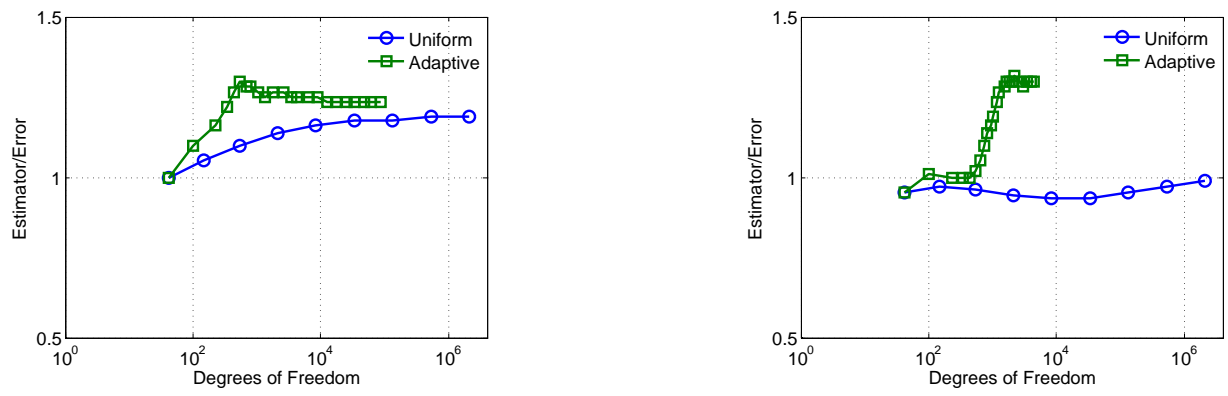

Figure 9: Example 2. Efficiency indices for $\left(\mathcal{R} \mathcal{T}_{0}, \mathcal{L}_{1}\right)$ with FEM and AFEM, for $\gamma=0.50$ (left) and $\gamma=0.25$ (right).
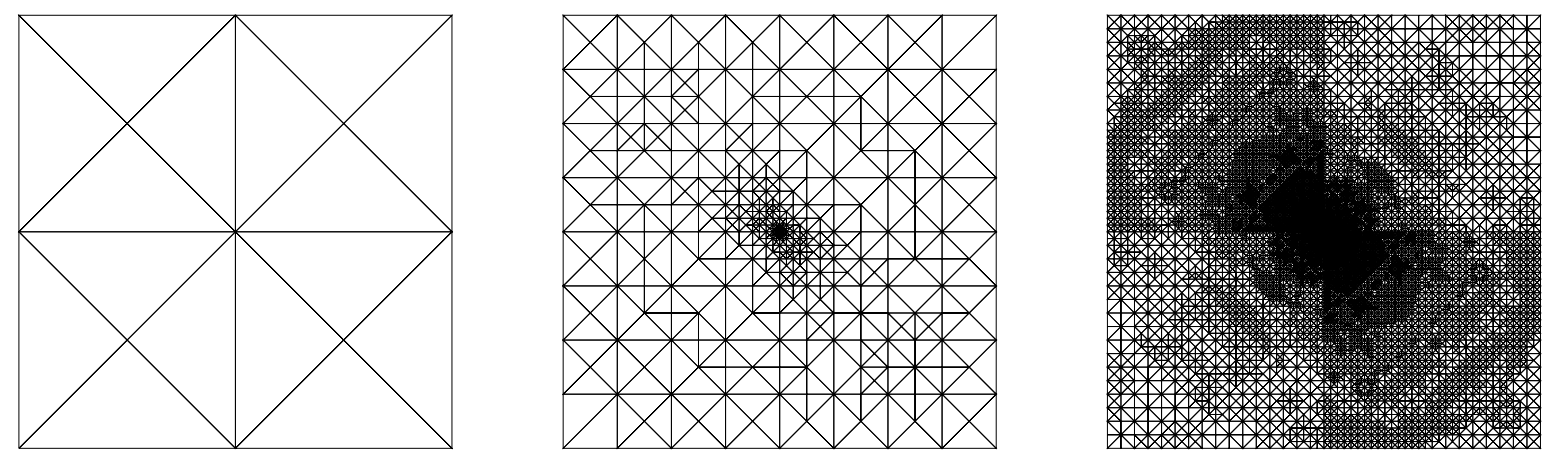

Figure 10: Example 2, $\gamma=0.50$. Adapted meshes obtained after 0, 10 and 20 AFEM iterations, composed of 16,880 and 24128 triangles, respectively.

In Figure 17 we show some meshes generated by AFEM. We can see there that the meshes are highly refined near the singularity points, $(-\epsilon,-\epsilon,-\epsilon)$ and $(1+\epsilon, 1+\epsilon, 1+\epsilon)$. Finally, in Figure 18 we show the pressure and the velocity fields obtained after 14 AFEM iterations (127578 tetrahedra). 

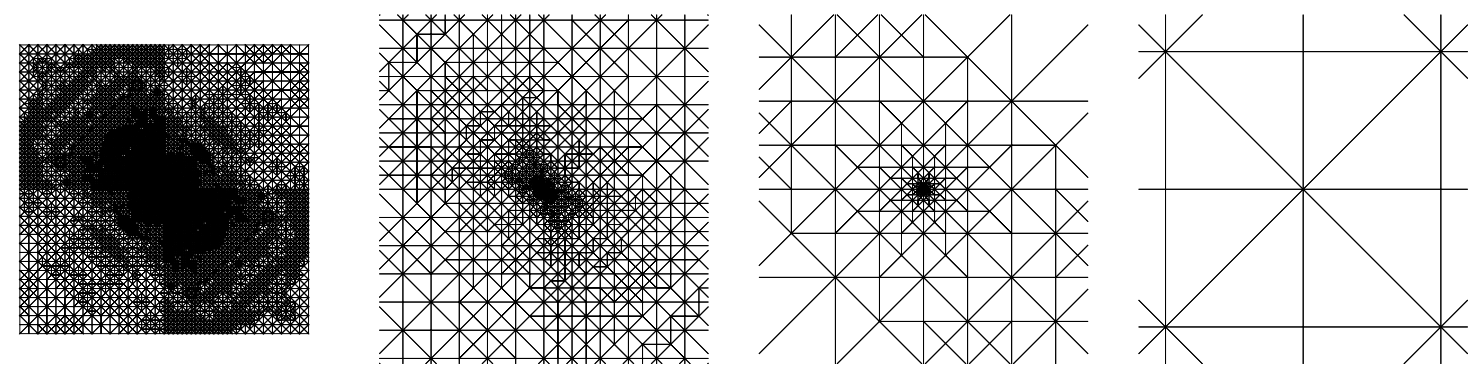

Figure 11: Example 2, $\gamma=0.50$. Adapted mesh obtained after 20 AFEM iterations (24128 triangles). From left to right: full mesh and zooms to $\left[-10^{-2}, 10^{-2}\right]^{2},\left[-10^{-4}, 10^{-4}\right]^{2}$ and $\left[-10^{-6}, 10^{-6}\right]^{2}$.
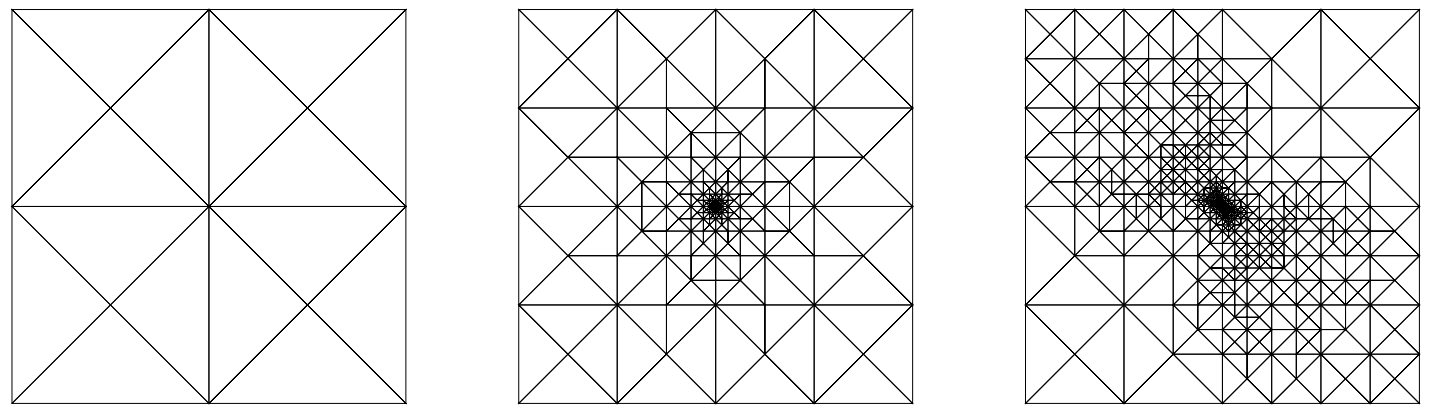

Figure 12: Example 2, $\gamma=0.25$. Adapted meshes obtained after 0, 10 and 20 AFEM iterations, composed of 16, 480 and 1744 triangles, respectively.
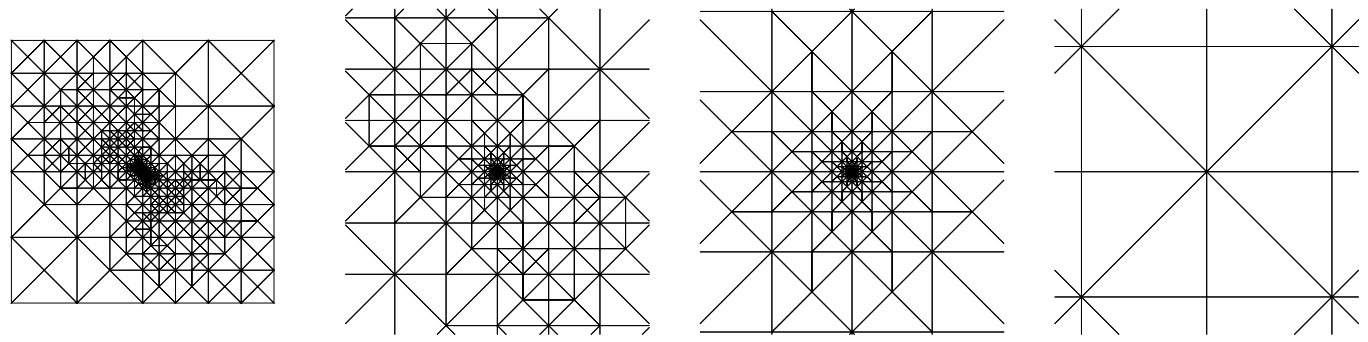

Figure 13: Example 2, $\gamma=0.25$. Adapted mesh obtained after 20 AFEM iterations (1744 triangles). From left to right: full mesh and zooms to $\left[-10^{-2}, 10^{-2}\right]^{2},\left[-10^{-4}, 10^{-4}\right]^{2}$ and $\left[-10^{-6}, 10^{-6}\right]^{2}$.

\section{Conclusions}

We considered a slight generalization of the method introduced by Masud and Hughes in [10] for the Darcy problem of porous media flow. The augmented variational formulation is obtained by 

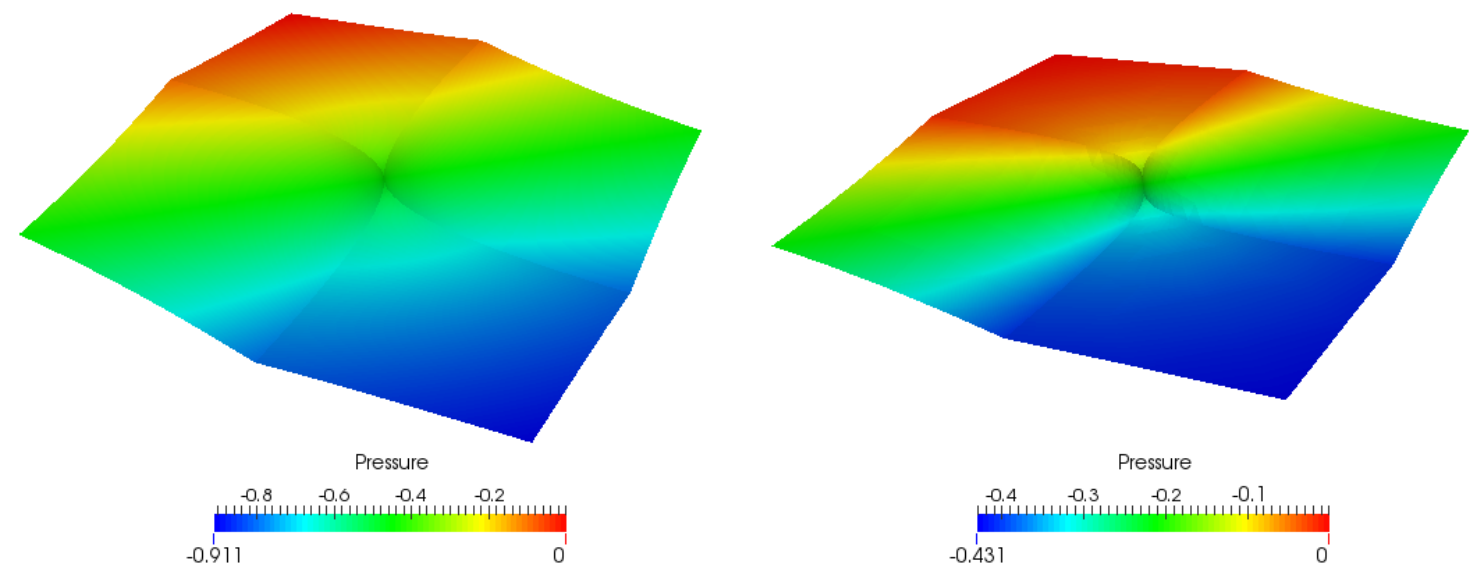

Figure 14: Example 2. Pressure obtained after 20 AFEM iterations for $\gamma=0.50$ (left) and $\gamma=0.25$ (right).

adding to the classical dual-mixed variational formulation two weighted residual type terms, that are related with Darcy's law and the mass conservation equation. We provided sufficient conditions on the stabilization parameters that ensure that the weak formulation is well-posed. Under these same hypotheses, we also proved that the corresponding Galerkin scheme is well-posed and a Céa-type estimate holds whatever finite-dimensional subspaces are used. In particular, we provide a priori error bounds when the fluid velocity is approximated by Raviart-Thomas or Brezzi-Douglas-Marini elements, and the pressure is approximated using continuous piecewise polynomials.

Our main contribution is the derivation of a two-term a posteriori error estimator of residual type for this discrete scheme. The two residual terms account for the error in Darcy's law and in the mass conservation equation. We remark that, besides the fact that it can be used with any finite element subspaces in $\mathbb{R}^{d}(d=2,3)$, this a posteriori error estimator is very easy to implement. Moreover, we proved that it is reliable and locally efficient. We remark that these theoretical results apply also to anisotropic porous media.

Finally, we implemented the method using the finite element toolbox ALBERTA. We showed that the method is robust with respect to the stabilization parameters. Numerical experiments also illustrate the good performance of the adaptive algorithm based on the a posteriori error estimator. Indeed, efficiency indices are close to one and the AFEM is able to localize the singularities and high-variation regions of the exact solution.

Acknowledgements. This research was partially supported by the Spanish Ministerio de Economía y Competitividad grants MTM2010-21135-C02-01, CGL2011-29396-C03-02 and CEN-20101010, by 

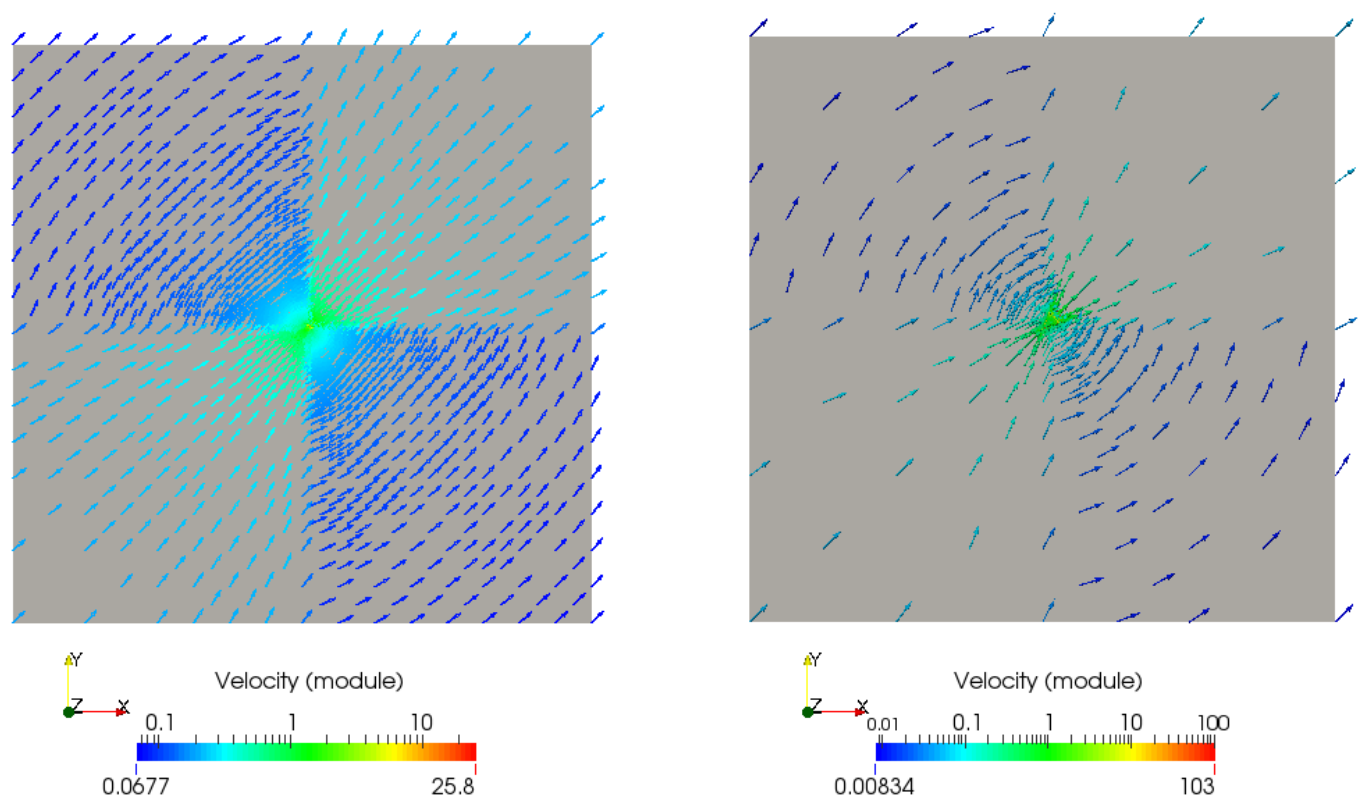

Figure 15: Example 2. Velocity field (log-scale) obtained after 16 AFEM iterations for $\gamma=0.50$ (left) and $\gamma=0.25$ (right).
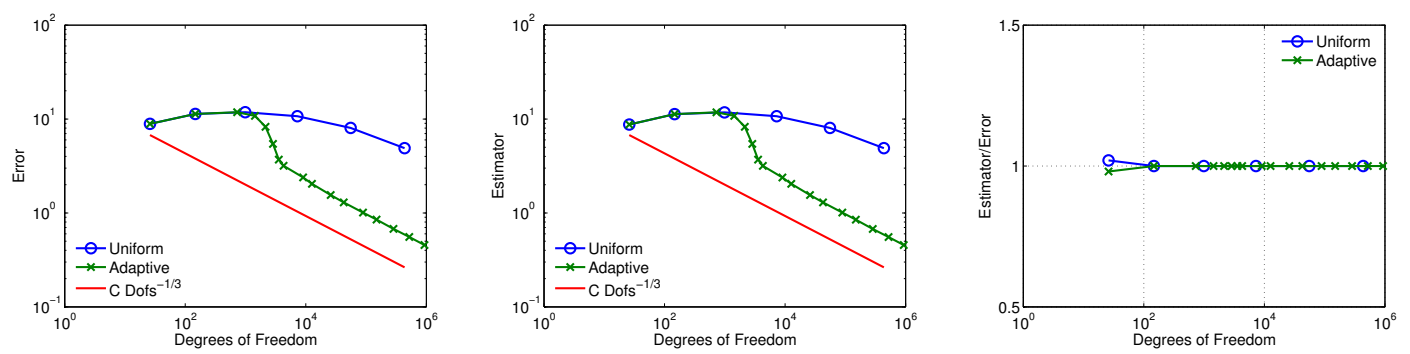

Figure 16: Example 3. Decays of total error (left) and estimator (center) and efficiency index (right) for $\left(\mathcal{R} \mathcal{T}_{0}, \mathcal{L}_{1}\right)$ with FEM and AFEM.

Consejería de Educación (Junta de Castilla y León, Spain) grant SA266A12-2 and by Dirección de Investigación of the Universidad Católica de la Santísima Concepción.

\section{References}

[1] F. Brezzi and M. Fortin, Mixed and Hybrid Finite Element Methods, Springer-Verlag, 1991. 

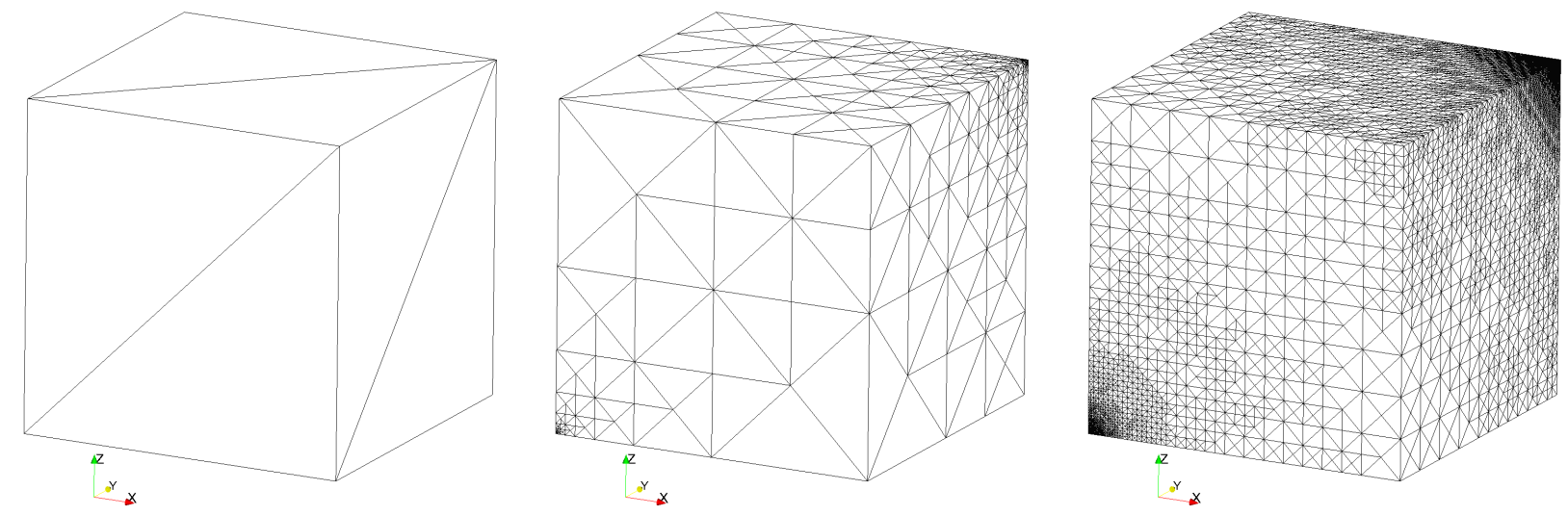

Figure 17: Example 3. Adapted meshes obtained after 0, 8 and 16 AFEM iterations, composed of 6, 3840 and 420390 tetrahedra, respectively.
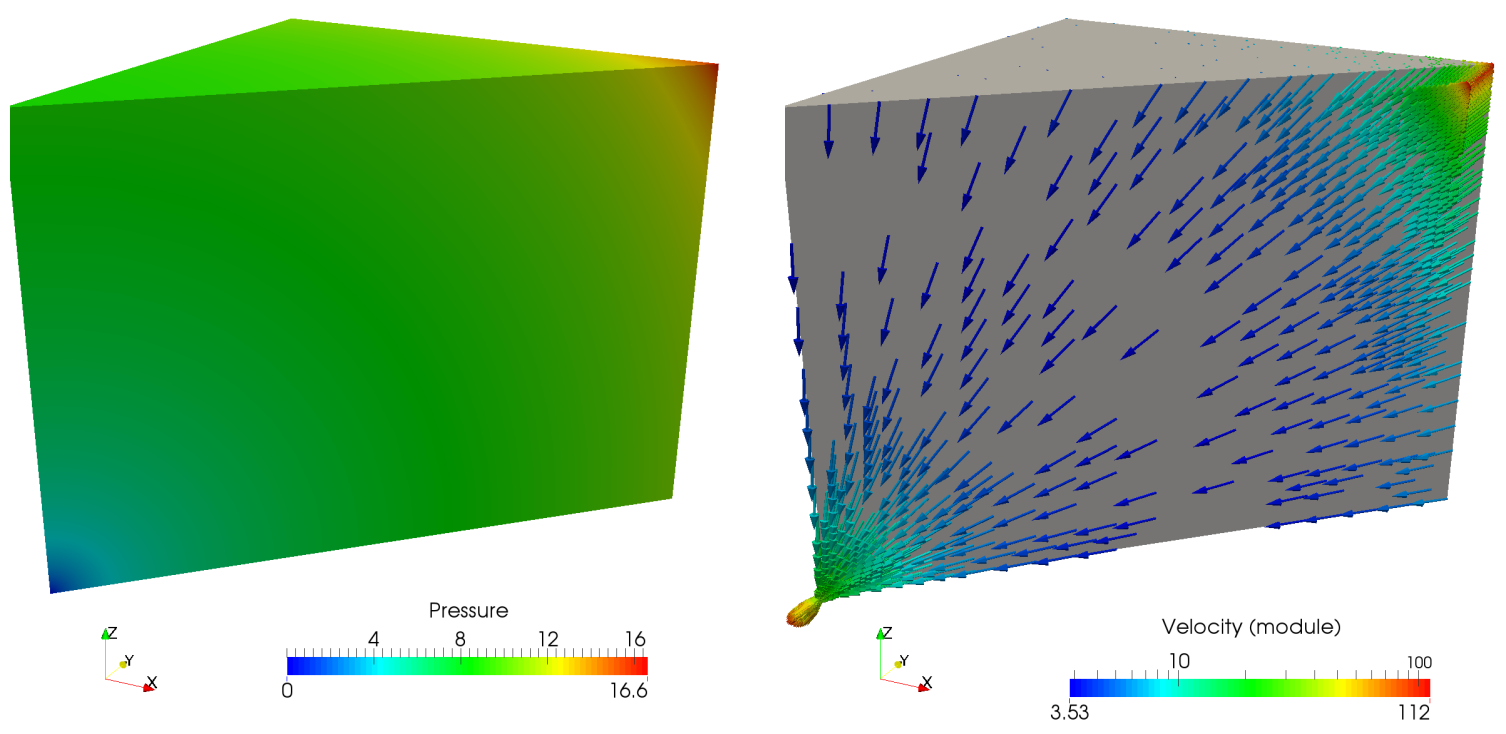

Figure 18: Example 3. Pressure and velocity field obtained after 14 AFEM iterations on a section of the domain $(0,1)^{3}$.

[2] J. M. Cascón, R. H. Nochetto and K. G. Siebert. Design and convergence of AFEM in H(div), Math. Models Methods Appl. Sci., vol. 17, pp. 1849-1881 (2007). 
[3] A.J. Chorin, Numerical solution of the Navier-Stokes equations, Math. Comput., vol. 22, pp. 745-762 (1968).

[4] H. Darcy, Les fontaines publiques de la ville de Dijon, Dalmont, Paris, 1856.

[5] J.W. Demmel, S.C. Eisenstat, J.R.Gilbert, X.S. Li and J.W.H. Liu, A super nodal approach to sparse partial pivoting, SIAM J. Matrix Analysis and Applications, vol. 20, pp 720-755 (1999).

[6] R. B. Kellogg, On the Poisson equations with intersecting interfaces, Applicable Anal. 4, 101$129,(1974 / 75)$.

[7] I. Kossaczky. A recursive approach to local mesh refinement in two and three dimensions, J. Comput. Appl. Math., vol. 55, no. 3, pp. 275288 (1994).

[8] M.G. Larson and A. Målqvist, A posteriori error estimates for mixed finite element approximations of elliptic problems, Numer. Math., vol. 108, pp. 487-500, (2008).

[9] S.M.C. Malta, A.F.D. Loula and E.L.M. García, Numerical analysis of stabilized finite element method for tracer injection simulations, Comput. Methods Appl. Mech. Engrg., vol. 187, pp. 119-136 (2000).

[10] A. Masud and T.J.R. Hughes, A stabilized mixed finite element method for Darcy flow, Comput. Methods Appl. Mech. Engrg., vol. 191, pp. 4341-4370 (2002).

[11] J.E. Roberts and J.-M. Thomas, Mixed and Hybrid Methods, in Handbook of Numerical Analysis, edited by P.G. Ciarlet and J.L. Lions, vol. II, Finite Element Methods (Part 1). NorthHolland, Amsterdam (1991).

[12] A. Schmidt and K. G. Siebert. Design of Adaptive Finite Element Software: The Finite Element Toolboox ALBERTA, LNCSE 42. Springer (2005).

[13] R. Temam, Une méthode d'approximation de la solution des équations de Navier-Stokes, Bull. Soc. Math. France, vol. 98, pp. 115-152 (1968). 